\title{
Efficient Editing of CSLD2 Orthologue by CRISPR/Cas9 Affects Cell Morphogenesis of Root Hair in Spinach
}

\author{
Yingping Cao \\ Chinese Academy of Sciences \\ Yue Xu \\ Chinese Academy of Sciences \\ Yue Zhang \\ Northeast Forestry University \\ Heng Zhang \\ Shanghai Normal University \\ Chen Bai \\ Shanghai Normal University \\ Zhi Qin \\ Shanghai Normal University

\section{Hailing Zhang} \\ Heilongjiang Academy of Agricultural Sciences

\section{Dongmei Ma} \\ Ningxia University \\ Quanhua Wang \\ Shanghai Normal University

\section{Meihong Sun} \\ Shanghai Normal University \\ Chunxiang Fu \\ Chinese Academy of Sciences \\ Shaojun Dai ( $\nabla$ daishaojun@shnu.edu.cn ) \\ Shanghai Normal University https://orcid.org/0000-0001-8063-6946
}

\section{Research Article}

Keywords: CRISPR/Cas9, cellulose synthase-like D (CSLD) gene, spinach, hairy root, root hair, transcriptomic analysis

Posted Date: January 7th, 2022

DOI: https://doi.org/10.21203/rs.3.rs-1180313/v1

License: () (i) This work is licensed under a Creative Commons Attribution 4.0 International License. Read Full License 


\section{Abstract}

CRISPR/Cas9 is a valuable tool and has been extensively employed to perform gene editing in plants. However, CRISPR/Cas9 has not been successfully used in spinach, an important leafy vegetable crop. Here, we precisely edited Spo23361 and Spo10340, two cellulose synthase-like D (CSLD) genes involved in root hair formation of spinach hairy roots, using CRISPR/Cas9 system. Four mutation types (i.e., replacement, insertion, deletion, and combined mutations) were observed, among which the deletion accounted for the vast majority (about 64.1\%). Mutation rate differed largely among different targets. Seven homozygous/bi-allelic and eight heterozygous/chimeric mutated lines of Spo23361 were obtained from 15 independent transgenic hairy root lines. All of the seven homozygous/bi-allelic lines displayed bulking and short root hairs, which exhibited the characteristics of Arabidopsis cs/d2 mutants. Thirteen heterozygous/chimeric mutated lines, but no homozygous/bi-allelic lines, of Spo10340 were obtained from 15 independent transgenic hairy root lines, all of which showed similar phenotype of root hair with normal hairy roots. The transcriptomic analysis further revealed that multiple gene expressions for cell wall modulation and membrane trafficking were disturbed, which might result in the inhibition of root hair growth in Spo23361 mutants. Our results indicate that Agrobacterium rhizogenes-mediated transformation using CRISPR/Cas9 is a simple and efficient genome editing tool in spinach. It lays a solid foundation for large-scale genome editing in spinach in future.

\section{Key Message}

Targeted mutagenesis of two CSLD genes (Spo23361 and Spo10340) by genome editing affect root hair formation of spinach hairy roots

\section{Introduction}

Spinach is an important nutritious green leafy vegetable, which is rich in carotenoids, folate, vitamin C, as well as calcium and other irons (Xu et al. 2017). We have published the draft genome sequence of a Chinese inbred spinach cultivar Sp75 (Spinacia oleracea, 2n=12) (Xu et al. 2017) and provided a primary $A$. rhizogenes-mediated transformation system of S. oleracea (Xu et al. 2019). We also have cultured new spinach varieties with higher stress (e.g., heat, salinity, and diseases) tolerance using traditional and molecular breeding strategies (Ge et al. 2017). Besides, the specific heat-responsive signaling and metabolic mechanisms in heat-tolerant spinach variety Sp75 and heat-sensitive Sp73 were reported using proteomics and phosphoproteomics methods (Zhao et al. 2018; Li et al. 2019). Although numbers of genes/proteins have been proposed to involved in transcription, calcium signaling, ROS homeostasis, endomembrane trafficking, and cross-membrane transport, their functions were not proved by molecular genetics, due to lack of a highly efficient gene editing system (Zhao et al. 2018; Li et al. 2019). Therefore, establishment of a gene editing system is critical for the investigation of molecular mechanisms in spinach development and stress response.

The clustered regularly interspaced short palindromic repeat (CRISPR)-associated protein (Cas9) system was firstly discovered as a component of the immune system from the bacterium Streptococcus thermophilus (Barrangou and Marraffini 2014). The DNA fragments of invading virus were caught by the host cell and integrated into the CRISPR loci. Then, the host cell used the transcribed RNA to guide the nuclease digesting the target site of virus transcript when the pathogenic organism infected again (Barrangou and Marraffini 2014). The simplified type II CRISPR/Cas9 system has been widely used in higher organisms. In this system, sgRNA could form a complex with Cas9 nuclease and guide the complex to target DNA sequence which can pair with the former 20 bases of sgRNA and was followed by an "NGG" sequence for the binding and cleavage of Cas 9 nuclease (Jinek et al. 2012; Ma and Liu 2016; Dong et al. 2017; Wang et al. 2018). Homologous recombination (HR) and non-homologous endjoining (NHEJ) were promoted in the higher organisms by the site-specific double-strand breaks generated Cas 9 nuclease which results in targeted mutagenesis.

In recent years, the type II CRISPR-Cas9 system through Agrobacterium tumefaciens transformation has become a powerful tool for investigating plant gene function and crop molecular breeding, but lacking of a mature transformation platform for some plants limited its applications (Gao 2021; Zhu et al. 2020). Fortunately, Agrobacterium rhizogenes-mediated transformation system is relatively easier to succeed in plants, because it induces the production of hairy roots rather than aims at germline transmission. It would be a satisfactory system after the regeneration from hairy roots was figured out in the coming future (Georgiev et al. 2012).

So far, CRISPR-Cas9 system has been successfully applied in hairy roots from many plant species for various purposes. For example, knocking out the committed diterpene synthase gene SmCPS1 by CRISPR/Cas9 in hairy roots from a traditional Chinese medicinal herb Danshen (Salvia miltiorrhiza) can completely inhibit the synthesis of various active ingredient tanshinones, such as cryptotanshinone, tanshinone IIA and tanshinone I (Bai et al. 2018). Besides, the CRISPR/Cas9 system-edited rosmarinic acid synthase gene SmRAS, functioned in the water-soluble phenolic acid biosynthetic pathway, obviously reduced the contents of various phenolic acids (i.e., rosmarinic acid and lithospermic acid B) in the hairy roots from S. miltiorrhiza (Zhou et al. 2018). In addition, CRISPR-Cas9 mediated-knockout of St16DOX gene caused the complete abolition of the accumulation of steroidal glycoalkaloids (SGAs) (i.e., alpha-solanine and alpha-chaconine) that showed the toxicity against various organism in potato hairy roots (Nakayasu et al. 2018). Besides of elucidating distinct reaction within a biosynthetic pathway, hairy root was considered as a transformative tool for interrogating gene function of root through CRISPR-mediated gene mutation, because type-specific expression of gene was similar in transformed hairy root by $A$. rhizogenes as compared with primary roots of $A$. tumefaciens transformants (Ron et al. 2014). In Brassica carinata, deletions in 
FASCICLIN-LIKE ARABINOGALACTAN PROTEIN 1 (BCFLA1) gene in the hairy roots resulted in shorter root hairs under inorganic phosphate deficient conditions, which proved a model role of hairy root system in rapidly assess gene function again (Kirchner et al. 2017).

To establish CRISPR/Cas9 system in spinach, we chose the established $A$. rhizogenes-mediated hairy root platform as a model. Interestingly, the hairy roots of spinach have abundant root hairs, which implied that the phenotype of root hairs could be taken as a better indicator for optimizing the CRISPR/Cas9- based gene editing system. Root hairs have important role in uptake of water and nutrients, and also decide the surface area between plants and soil microbes (Libault et al. 2010). Root hairs are tip-growing and tubular outgrowths of specialized root epidermal cells, which needs rapid cell wall assembly in the tips. The deficient and/or disrupted cell wall components lead to root hairs deformed and even ruptured (Yin et al. 2011). A number of genes involved in the cell wall modulation during hair tip growth. Cellulose synthase-like D (CSLD) gene belongs to a subfamily of cellulose synthase-like (CSL) gene family, which functions in the cellulose synthesis in cell wall of root hairs (Bernal et al. 2008). It has been reported that, among six AtCSLDs, AtCSLD2 and AtCSLD3 were required for normal root hair growth in Arabidopsis (Bernal et al. 2008; Yin et al. 2011; Qi et al. 2013). AtCSLD3 was involved in the biosynthesis of $\beta$-glucan-containing polysaccharides in cell wall for root hair elongation (Favery et al. 2001), while more AtCSLD2 was required during late stage of hair development than AtCSLD3 (Bernal et al. 2008). The cs/d2 mutant exhibited abnormal root hair and many root hairs bulging and rupturing late during root development process (Bernal et al. 2008).

In the present study, SpCSLD2 (Spo10340) and SpCSLD3 (Spo23361) were chosen as the target genes edited by CRISPR/Cas9 system in hairy roots of spinach. The abnormal root hairs were observed in homozygous/biallelic mutated lines, and the transcriptome analysis provide the signal and metabolic mechanisms in the CRISPR/Cas9- edited hairy roots of spinach. To our knowledge, this is the first report for the CRISPR/Cas9 system applied in spinach.

\section{Materials And Methods}

\section{Plant materials, growth and culture conditions}

Chinese inbred spinach cultivar Sp75 was used in this study (Xu et al. 2017). The seeds were surface-satirized with 75\% ethyl alcohol and 5\% sodium hypochlorite (Xu et al. 2019). All the sterile seeds were germinated on solid $1 / 2 \mathrm{MS}$ basal medium containing $30 \mathrm{~g} / \mathrm{L}$ sucrose and $10 \mathrm{~g} / \mathrm{L}$ agar, and cultured under $26^{\circ} \mathrm{C}$ and $16 \mathrm{~h}$ light/ $8 \mathrm{~h}$ dark. The explants from leaves were used to induce hairy roots. The different tissues and organs from two-month-old seedlings were used for qRT-PCR analyses. Two-month-old hairy roots from normal wild type (WT) and Spo23361 mutant (line 3) were collected for transcriptomic analysis.

\section{Bioinformation analysis of CSLD protein sequence}

The five SoCSLDs gene sequences were achieved by BLAST using the AtCSLD3 gene as query sequence in the website (http://www.spinachbase.org/). Multiple alignment analysis of the full-length protein sequences was performed by Clustal X program. The phylogenetic tree was constructed with MEGA 3.1 program using the Neighbor-joining (NJ) method with 1,000 bootstrap replicates. The pl and molecular weight (MW) of Sp23361 and Sp10340 were predicted in the website (https://web.expasy.org/compute_pi/). The conserved domains of Spo23361 and Spo10340 were online analyzed by the Conserved Domain Database (CDD) of NCBI (https://www.ncbi.nlm.nih.gov/cdd).

\section{Quantitative real-time PCR}

Total RNAs were extracted from stem, leaf, flower, stipe, root and hairy root using Trizol reagent (Invitrogen Corporation). RT-PCR was carried out using 500 ng of total RNA with PrimeScript ${ }^{\text {TM }}$ RT reagent Kit with gDNA Eraser (TaKaRa) following the manufacturer's protocol. Gene expression was assayed using an Rroche LightCycler480 real-time PCR System. The expression of actin gene (Spo23599) was used as an internal control. The primers used to amplify transcripts were listed in Table S8.

\section{Target sites design and vector construction}

The specific sequences of Spo23361 and Spo10340 were amplified by two pairs of primers in Table S8, respectively. Three target sites were selected for each gene according to Ma et al. (2016) (Fig. S1A,1B). Briefly, the 20 bp sequence before the NGG region should have appropriate GC content ( $\geq 30 \%$ ), avoid four consecutive T nucleotides, and exclude the target candidates that form the hairpins of longer than 6 bp with sgRNA sequence. The tRNA-gRNA cassette strategies were used (Xie et al. 2015). Cas 9 was driven by the CaMV 35 S promoter. Target sequences separated by tRNA and followed by one gRNA were driven by $A$. thaliana U3b promoter. Bargene driven by CaMV 35S promoter was used as a selective marker (Fig. S1c).We constructed a modified pYLCRISPR/Cas9 35s-B (GenBank accession number: Al133729.1) vector. The segment of two Bsa I cutting sites and CCDB in the vector was replaced with the sequence containing a AtU3b snRNA promoter, two new Bsa I cutting sites, and a sgRNA sequence. AtU3b snRNA promoter was amplified from pYLsgRNA-AtU3b (GenBank accession number: KR029097.1) (Fig. S1d). The tRNA-gRNA cassette was linked to the constructed vector according the methods of Ma et al. (2016) and Xie et al. (2015). The fragments containing tRNA and/or sgRNA sequence were amplified from pGTR vector (Beijing Genomics Institute) using the primers listed in Table S8. The mixed fragments and the modified pYLCRISPR/Cas9 35s-B vector, were digested by Bsa I and linked by T4 ligase in one step (Ma et al. 2016; Xie et al. 2015). The CRISPR/Cas9 vector for Spo23361 and Spo10340 containing three target sequences, and tRNA-gRNA expression cassette was transferred into A. rhizogenes strain LBA9402 for subsequent experiment. 
A. rhizogenes strain LBA9402 carrying the CRISPR/Cas9 vector for Spo23361 and Spo10340 was used for the gene transfer experiment. The Agrobacterium strain was cultured by shaking in liquid LB containing $50 \mathrm{mg} / \mathrm{L}$ kanamycin and $20 \mathrm{mg} / \mathrm{L}$ rifampicin at $28{ }^{\circ} \mathrm{C}$ and under $200 \mathrm{rpm}$. The $\mathrm{OD}_{600}$ of 0.6- 0.8 was the proper concentration for inoculation. The bacterial liquids were collected and re-suspended to $\mathrm{OD}_{600}$ of 0.2 using liquid $\mathrm{MS}$ medium containing $100 \mu \mathrm{M}$ acetosyringone. The leaf discs $(\sim 0.5 \mathrm{~cm} \times 0.5 \mathrm{~cm})$ taken from the 30-day-old aseptic seedlings were submerged in $A$. rhizogenes suspension for $10 \mathrm{~min}$, and then co-cultured for two days in darkness. The discs were sub-cultured in a selection medium (SH base medium containing $1 \mathrm{mg} / \mathrm{L}$ phosphinothricin and $300 \mathrm{mg} / \mathrm{L}$ timentin). Hairy roots ( $2 \mathrm{~cm}$ in length) were cut from the discs and sub-cultured in the selection medium for further identification.

The genomic DNA of the putatively transformed plants was extracted using CTAB method. In order to identify the co-transformed hairy root lines, PCR analyses were performed to detect the presence of bar, Cas 9 and ro/B genes using primers in Table S8.

\section{Mutation type analysis}

Fragments containing the target sites were amplified using the primer in Table S8. The PCR products were sequenced to detect mutagenesis. The PCR products with mixed peaks and/or sequence changes were purified with DNA Purification Kit and ligated in the pMD19-T easy vector. The ligated products were transformed into Escherichia coli strain Top10, and five positive clones of each transgenic line were sequenced to analyze their specific mutation types.

\section{Phenotype observation}

The phenotype of root hairs was observed under a microscope OLYMPUS SZX16. The length and density of root hairs were measured from three lines of mutants and normal hairy roots, respectively $(n=8)$.

\section{Off-target analysis}

The potential off-target sites were predicted according the method of (Li et al. 2017). Spinach genome was downloaded from website (http://www.spinachbase.org/). The putative off-target locus were blasted among the whole genome of spinach. The sites containing no more than 3-bp mismatches in the 20-bp target sequence were taken as potential off-target sites (Table S2). We amplified the sequence containing the potential off-target sites by PCR using gene-specific primers (Table S8). The PCR products were sequenced to verify the occurrence of mutation.

\section{Transcriptome sequencing of root hairs}

Total RNA was extracted from hairy root of normal wild type (control) and Spo23361 mutants using the mirVana miRNA Isolation Kit (Ambion). The samples with RNA Integrity Number (RIN) $\geq 7$ were subjected to the subsequent analysis. The cDNA library was constructed using TruSeq Stranded mRNA LTSample Prep Kit (Illumina, San Diego, CA, USA), and the libraries were sequenced on the Illumina sequencing platform (HiSeqTM 2500 or Illumina HiSeq X Ten) according to the method of Xu et al. (2017). 125bp/150bp paired-end reads were generated. The transcriptome de novo assembly was carried out using Trinity (http://trinityrnaseq.sourceforge.net). The clean reads were mapped to spinach reference genome using hisat2.

Fragments Per Kilobase of transcript per Million (FPKM) of each gene and read counts value of each transcript (protein_coding) were calculated using bowtie2 and eXpress, respectively. The analysis of differential expression gene (DEG) among samples was performed using the DESeq (2012) $\mathrm{R}$ package. The $p$ value $<0.05$ and Fold Change $>2$ (or $<0.5$ ) were applied to evaluate the difference significance. Hierarchical cluster of DEGs was performed to explore transcripts expression pattern. GO and KEGG pathway enrichment of DEGs were performed using R based on the hypergeometric distribution.

Heatmap was plotted using an OmicShare tool (http://www.omicshare.com/tools/). The KEGG pathway, as well as GO biological processes and cellular component enrichment analyses were conducted using the Metascape analysis (http://metascape.org/). Terms with a $p$-value < 0.01 , a minimum count of 3 , and an enrichment factor (the ratio between the observed counts and the counts expected by chance) $>1.5$ were collected and grouped into clusters on the bases of their membership similarities.

Protein-protein interaction between DEG encoding proteins were predicted by the online tool STRING 10 (http://string-db.org/) with the minimum required interaction score set as $>0.4$. All other parameters were set as defaults, and all active prediction methods were used. The network was visualized using Cytoscape 3.7.1 software (http://www.cytoscape.org/). All the identification (ID) input for the interaction network analyses were protein homologs in Arabidopsis thaliana, which were found by sequence BLASTing in the TAIR database. Proteins (nodes) were shown as bubbles whose color gradient from light blue to dark green according to the node degree distribution. The related interactions (edges) between proteins were shown as gray lines, whose width represented the strength of interaction score. The function categories were represented by different colors of bubbles in network and columns.

\section{Results}


Five spinach genes, Spo23361, Spo10340, Spo16352, Spo12722 and Spo16366, were predicted from the spinach (S. oleracea) genome based on the similarity to the Arabidopsis AtCSLD3 gene (Favery et al. 2001). Phylogenetic analysis of the spinach and Arabidopsis CSLDs indicated that the five spinach genes clustered with different Arabidopsis CSLD genes respectively. Among them, Spo23361 and Spo10340 were clustered with AtCSLD2 and AtCSLD3 together (Fig. 1a). The open reading frames of Spo23361 and Spo10340 have 3,444 and 3,459 nucleotides, respectively. They encode two putative proteins containing 1,447 and 1,152 amino acid resides with similar molecular mass (128,557 Da and 130,011 Da) and pl (6.9 and 7.06), respectively. Pairwise comparison analysis showed that Spo23361 and Spo10340 exhibit $80 \%$ and $81 \%$ identity to both AtCSLD2 and AtCSLD3 (Fig. 1b), respectively. Conserved domain analysis showed Spo23361 and Spo10340 proteins have the conserved DDDQ/RxxRW motif (Richmond and Somerville 2000), indicating they belong to the CSL family (Fig. 1b).

The expression of five SoCLSD genes were examined in stems, roots, leaves, petioles, and flowers from two-month-old seedlings of spinach, as well as in hairy roots induced from leaves by A. rhizogenes strain LBA9402 using qRT-PCR analysis. Spo23361 and Spo10340 expressed in all these organs with similar patterns, and both of them showed obviously higher expressions in roots and hairy roots, respectively (Fig. 1c). Especially, the expression of Spo23361 was significantly higher in both roots and root hairs than that in other organs/tissues (Fig. 1c). In addition, the others (Spo16352, Spo12722, and Spo16366) were also highly expressed in roots.

All these implies that Spo23361 and Spo10340 would be good candidates for investigating the function of AtCSLD2 and AtCSLD3 homologues in root hair development of spinach using CRISPR/Cas9 gene editing system.

CRISPR/Cas9 vector construction and co-transformation efficiency in hairy roots

Partial sequences of Spo10340 and Spo23361 were amplified from cDNA of roots. Three targets were designed for each gene. The tRNA-gRNA cassette strategies were used to construct the CRISPR/Cas9 vector (Fig. S1) (Xie et al. 2015).

T-DNA of Ti plasmid harboring CRISPR/Cas9, tRNA-gRNA and bar expression cassette were transferred into leaf explants of spinach using $A$. rhizogenes strain LBA9402. Hairy roots appeared after 10-20 days of infection, which looked very similar with normal roots of spinach (Fig. 2a, b). Numerous independent transgenic hairy roots were produced for each construct (22 root lines for Spo10340, and 16 for Spo23361) (Fig. 2c, d). The PCR detection implied that rolB, Cas9, and bargenes were successfully transformed into the spinach, and about $80 \%$ hairy root lines ( 30 from 38 lines) were co-transferred into Ri T-DNA and Ti T-DNA (Fig. 2c, d).

\section{Genotyping and phenotyping of edited hairy root lines of Spo26631}

Total 15 hairy root lines of Spo26631 edited by CRISPR/Cas9 system were obtained (Fig. 3 and Table S1), among of which seven lines (i.e., line 2, line 3 , line 4, line 8, line 12, line 13, and line 14) showed obvious phenotypes of short root hair (Fig. 3a, b). Besides, the abnormal middle and/or top of root hairs from these lines looked like bulging balls (Fig. 3a, b). Sequence analysis showed that all of seven lines were bi-allelic/homozygous mutated lines with edited sequence in the same or different target sites in two sister chromatids (Fig. 3e and Table S1). The root hair density of seven lines was not changed compared with that in normal hairy roots (Fig. 3f). However, the lengths of root hairs of seven lines were significantly reduced to the one twentieth of normal length (Fig. $3 \mathrm{~g}$ ). Another eight co-transferred lines were also sequenced. Among them, five heterozygous mutated lines (i.e., line 5, line 6, line 7, line 9, and line 11) contained one mutation type in each line, two chimeric mutated lines (line 1 and line 10) had three or two mutation types in each line, and the line 8 had a wide-type genotype in three target sites (Table S1). All of the heterozygous or chimeric mutated lines showed no difference in the length and density of root hairs when compared with normal hairy roots (Fig. 3c-g).

\section{Genotyping and phenotyping of edited hairy root lines of Spo10340}

Twenty-two hairy root lines were obtained for the gene Spo10340, among of which 15 lines were found co-transferred with rol B and Cas 9 genes (Fig. 2d). No lines showed obviously stunted root hairs. The 15 lines were submitted to target sequence analysis. Among them, 13 lines (line 1 to line 13) were edited in more than one target sites, the remaining 2 lines had no change at the target sites. However, no bi-allelic and homozygous mutants were recovered. All of them were either chimeric or heterozygous lines (Fig. 4a-d and Table S1). The density and the length of root hairs were similar with those of normal hairy roots (Fig. 4 e, f).

\section{Mutation variety and frequency of CRISPR/Cas9- edited hairy roots}

Fifteen co-transferred hairy root lines of Spo23361 and Spo10340 were performed PCR analyses, and their PCR amplicons with the target sites were sequenced. Among them, fourteen lines of Spo23361 and thirteen lines of Spo10340 showed mixed peaks, indicating that these lines were edited by CRISPR/Cas9 at the target sites (Table 1). The PCR amplicons from the 27 hairy roots were further confirmed by TA cloning and sequencing (Table S1). The editing efficiency varies widely among the three targets of each gene of Spo23361 and Spo10340 (Table 1). The third target site of Spo23361 and the first target site of Spo10340 showed the highest mutation rate of $86.7 \%$. Four mutation types were observed, including replacement, insertion, deletion and combined mutations (i.e., more than one mutation type in one target site). For Spo23361, the insertion, deletion, and combined mutations occurred at the third target site, the replacement and deletion mutations occurred at the first target site, and only replacement mutation occurred at the second target site (Table 1). Interestingly, almost all the target sites except the first and second target of 
Spo23361 showed that the editing mode of deletion accounted for the majority. For Spo10340, four mutation types appeared at the first target site, while two mutation types (deletion and combined mutation) appeared at the second and third target site (Table 1).

Table 1

Detailed information of the mutation types of different targets in Spo23361 and Spo10340.

\begin{tabular}{|c|c|c|c|c|c|c|c|c|c|c|}
\hline \multirow[t]{2}{*}{ Gene } & \multirow{2}{*}{$\begin{array}{l}\text { No. } \\
\text { of } \\
\text { hairy } \\
\text { root } \\
\text { lines }\end{array}$} & \multirow{2}{*}{$\begin{array}{l}\text { No. of } \\
\text { hairy } \\
\text { root } \\
\text { lines } \\
\text { with } \\
\text { mutation }\end{array}$} & \multirow[t]{2}{*}{ Target site/sequence } & \multirow{2}{*}{$\begin{array}{l}\text { GC } \\
\text { content } \\
(\%)\end{array}$} & \multirow{2}{*}{$\begin{array}{l}\text { No. of } \\
\text { hairy } \\
\text { root } \\
\text { lines } \\
\text { with } \\
\text { mutation } \\
\text { at } \\
\text { different } \\
\text { targets }\end{array}$} & \multirow{2}{*}{$\begin{array}{l}\text { Mutation } \\
\text { rate }(\%) \\
\text { at } \\
\text { different } \\
\text { targets }\end{array}$} & \multicolumn{4}{|c|}{ Types of mutation (\%) } \\
\hline & & & & & & & $\mathrm{i}$ & $r$ & $d$ & c \\
\hline \multirow[t]{3}{*}{ Spo23361 } & 15 & 14 & 1/GTGTCAAACTCССТCTTTAC & 45 & 3 & 20.0 & 0 & $66.66 \%$ & $33.33 \%$ & 0 \\
\hline & & & 2/СTCCTACCCAGCAGAGACGA & 60 & 1 & 6.7 & 0 & $100 \%$ & 0 & 0 \\
\hline & & & 3/AAACCAGAGTTCACAAACCA & 40 & 13 & 86.7 & $4.76 \%$ & 0 & $80.95 \%$ & $14.28 \%$ \\
\hline \multirow[t]{3}{*}{ Spo10340 } & 15 & 13 & 1/AACATCCAATGAGCAGGAGT & 45 & 13 & 86.7 & $23.07 \%$ & $7.68 \%$ & $42.30 \%$ & $26.92 \%$ \\
\hline & & & 2/ACAAAGGGGACATATGGGTA & 45 & 9 & 60.0 & 0 & 0 & $72.22 \%$ & $27.77 \%$ \\
\hline & & & 3/TTCTGGGATTCTTCCTCGTG & 50 & 4 & 26.7 & 0 & 0 & $85.71 \%$ & $14.28 \%$ \\
\hline
\end{tabular}

i: insertion, d: deletion, r: replacement, c: combined mutation (more than one mutation type in one allele).

To detect the off-target events in CRISPR/Cas9 edited hairy roots of spinach, potential off-target loci following protospacer adjacent motif (PAM) sequences were predicted using the program written by Li et al (2017). They were highly homologous and not more than three mismatches of each target sequence in the hairy roots of Spo23361 and Spo10340 (Table S2). Only one off-target locus was predicted in the first and second target sequence of Spo23361, respectively. However, five off-target locus were found in the third target sequence of Spo23361 (Table S2). For Spo10340, there were one and two off-target sequences in the first and second target sequence, respectively. To examine whether these predictions happened in our results, 15 hairy root lines of Spo23361 and Spo10340 were submitted for sequencing, respectively, and no site mutation was detected (Table S2).

\section{Transcriptomic analysis of CRISPR/Cas9 edited hairy roots}

To evaluate the differentially-expressed genes (DEGs), the transcriptomics analyses of Spo23361 mutants and normal hairy roots were performed by using RNA-seq based on Illumina platform. A total of 25,495 unique genes were identified and quantified, 305 of which were more than 2.0-fold change $(p<0.05)$ in abundance and considered as DEGs in hairy roots of Spo23361 mutant when compared with normal hairy roots. The hierarchical clustering indicated that 305 DEGs were clustered into two main groups (Figure 5a and Table S3). Metascape analysis showed that 13 GO biological processes of the corresponding genes were enriched (Figure 5b, c and Table S4). Cluster I showed a significantly- reduced pattern of 169 DEGs in Spo23361 mutants. The 57 reduced DEGs in Cluster I were enriched in $13 \mathrm{GO}$ biological processes including biosynthesis of salicylic acid, phenylpropanoid and flavonoid, response to salicylic acid, iron ion, bacterium, and hypoxia, metal ion and intercellular transports, defense response, protein folding, as well as cell death (Figure 5b, c and Table S4). Besides, 136 DEGs in Cluster II were induced in Spo23361 mutants. Among them, 32 genes were enriched in response to various stresses (e.g., salicylic acid, iron ion, hypoxia, and bacterium), ion transport, cell death, as well as biosynthesis of flavonoid and phenylpropanoid (Figure 5b, c and Table S4). Interestingly, DEGs were highly enriched in several clusters, including the metal ion transport (blue node), salicylic acid biosynthesis (yellow node), hypoxia (green node), flavonoid biosynthesis (purple node), and host interaction and intercellular transport (orange and dark green nodes) (Figure 5b and Table S4). In addition, these DEGs were enriched in several cellular components including cell wall, cell periphery, extracellular region, plasmodesma, plasma membrane, vacuole, and whole membrane (Figure 5d and Table S5), which indicated the cell wall modulation and membrane trafficking were seriously disturbed in the Spo23361 mutants.

To predict the relationship of DEGs in mutant roots, the Arabidopsis homologs of spinach proteins encoding by DEGs were found by sequence BLASTing in TAIR database (Table S6), and then were subjected to the web-tool SRING 10 for predicting protein-protein interaction (PPI) networks. The network showed 116 nodes (represent proteins encoding by DEGs) with 172 node degrees (represent PPIs) in mutants (Figure 5e and Table S7). The node colors with gradient from light blue to dark green indicated the node degree distributions (Figure 5e and Table S7). In this network, the dark green nodes represented cinnamyl alcohol dehydrogenase 3 , calmodulin-binding protein $60-\mathrm{C}$, receptor-like protein kinase, and $70 \mathrm{kDa}$ heat shock protein have more than 10 node degrees for each, implying their complicated interaction with other proteins. Interestingly, the nodes representing proteins encoding by DEGs for cell wall metabolism, protein folding and processing, membrane and transport, as well as signaling have more node degree than other nodes, suggesting that these signal and metabolic processes were obviously affected in mutants (Figure 5f, $g$, and Table S7). The transcriptomic results indicate that the gene expression patterns were significantly disturbed in Spo23361 mutants and might affect the root hair phenotype. 


\section{Discussion}

\section{Spinach Spo23361 was predicted to be the homologous gene of AtCSLD2}

In Arabidopsis, CSLD family has six members encoding glycosyltransferases that are responsible for the synthesis of the glycan backbones of cellulose and most polysaccharides (Richmond and Somerville 2000). The six AtCSLDs regulate the growth of stems, roots, and pollen tubes (Bernal et al. 2008; Wang et al. 2011; Yoo et al. 2012). Among them, CSLD5 is necessary for stem growth, and Arabidopsis atcs/d5 mutants showed stem growth defects (Bernal et al. 2008). AtCSLD1 and AtCSLD4 participate in maintaining tip-growth of pollen tubes for efficient transmission of male gametes (Bernal et al. 2008; Wang et al. 2011), whereas AtCSLD2 and AtCSLD3 are required for proper root hair growth (Yoo et al. 2012 ). AtCSLD3 encodes a distinct (1-4)- $\beta$-glucansynthase, which is critical for apical plasma membranes upon tip-growth of root hairs (Park et al. 2011). The root hairs of atcs/d3 mutants ruptured immediately after the initiation, which caused a fewer hair phenotype in roots (Favery et al. 2001; Wang et al. 2001). The root hairs of atcs/d2 mutant exhibited various types of deformities like severely protruding at the tip and the base area of the root hairs (Bernal et al. 2008). Moreover, atcs/d2 root hairs ruptured at a later stage when considerable hair growth had already initiated, and could resume growth after rupturing in some cases, resulting in bulking and short root hair phenotypes in atcsld2 mutants (Bernal et al. 2008; Yin et al. 2011; Yoo et al. 2012).

In our results, the analyses of phylogenetic tree and homology alignment indicated that both Spo23361 and Spo10340 from five SpCLSDs were the homologs of AtCSLD3 and AtCLSD2 (Fig. 1a). Besides, we found that Spo23361 and Spo10340 were highly expressed in roots and hairy roots of spinach (Fig. 1c), which were similar with the highest expression of AtCSLD3 and AtCLSD2 in Arabidopsis roots (Bernal et al. 2008). Interestingly, the two poplar genes, PdCSLD5 and PdCSLD6, also have higher similarity with AtCSLD2 and AtCSLD3, which were grouped into one branch in the phylogenetic tree (Qi et al. 2013). It has been found that overexpression of PdCSLD5 and PdCSLD6 can rescue the root hair defect phenotype of atcsld3 mutant, suggesting that PdCSLD5 and PdCSLD6 are the homologous genes of AtCSLD3 (Qi et al. 2013).

In our study, all the bi-allelic or homozygous mutants of Spo23361 gene generated by CRISPR/Cas 9 system exhibited short and/or bulking root hairs. However, the root hair numbers were not changed compared with the wide type (Fig. 3). Importantly, the phenotype of these lines was stable during the four-month subculturing process. This indicates that Spo23361 has similar function of AtCSLD2 rather than AtCSLD3. However, in our study, all these CRISPR/Cas9- edited lines of Spo10340 were either chimeric or heterozygous, leading to no stable root hair phenotype observed (Fig. 4). Even so, we still tried our best to repeat this experiment for obtaining bi-allelic or homozygous mutated lines for Spo10340 genes. Unfortunately, there was still no lines showing the similar phenotype with atcsld3 mutant, although 17 co-transferred lines were induced (data not shown). Therefore, we suspect that the homozygous or bi-allelic mutation of $S p 010340$ resulted in thorough growth inhibition of hairy roots, which is similar with the phenotype of root hair loss in atcsld3 mutants (Wang et al. 2001). However, the homolog relationship of Spo10340 and AtCSLD3 still needs to be further investigated.

\section{The tRNA-target-gRNA cassette expression system was an efficient tool for multiply targeted genome modification in spinach}

In this study, we tested the use of plant-optimized Streptococcus pyogenes CRISPR-associated protein 9 (SpCas9) and AtU3b promoter to selectively modify the spinach genome. Our results revealed that 27 from 30 hairy root lines were edited successfully, indicating that the SpCas 9 and AtU3b promoter worked efficiently in spinach (Table 1). All the six target sites of Spo23361 and Spo10340 were edited to diverse mutation types, including replacement, insertion, combined mutation, and deletion, and the highest mutation frequency reached to $86.7 \%$ (Table 1). The high editing rate was resulted from the tRNA-gRNA cassette expression system and multiple target sequences for each gene, since tRNA could not only serve as enhancer for AtU3b but also as a "divider" for the sgRNAs.

The mutation frequency of different target was ranged from $6.7-86.7 \%$ in our results, which was probably affected by various factors. It was reported that GC percentage of overall targets (Gagnon et al. 2014) and the 6-PAM-proximal nucleotides (Ren et al. 2014) are positively associated with editing efficiency in zebrafish (Danio rerio) and Drosophila, respectively. In the present study, the GC contents of the six target sites were range from $40-60 \%$, which was reported to be favored for efficient on-target cleavage (Liu et al. 2016). For Spo23361, the GC content of the 1st, 2nd, and 3rd target sequence was $45 \%, 60 \%$ and $40 \%$, while the GC content of the 1 st, 2 nd, and 3 rd target sequence of Spo 10340 was $45 \%$, $45 \%$ and $50 \%$, respectively. However, the highest mutation rate was occurred in the 3rd target site of Spo23361 and the 1st target sequence of Spo10340, which have the lowest GC content both in the target sequences (40\% and $45 \%$ ) and in the 6-PAM-proximal nucleotides (2/6 and $3 / 6)$. This is not consistent with the previous notion (Gagnon et al. 2014; Ren et al. 2014; Liu et al. 2016). In fact, it has been reported that the modulation of mutation efficiency of CRIPSR/Cas9 system was sophisticated and finetuned by diverse factors, such as the promoter used to drive sgRNA expression (Zhou et al. 2018), the efficiency of gRNA to search and target to the specific site (Johnson et al. 2015), the T-DNA insertion site in genome (Shan et al. 2013b), as well as the transformation method (Oh et al. 2020). In addition, the mutation ratio of CRIPSR/Cas9 system was variable in different plant species (Feng et al. 2013; Cai et al. 2015; Fauser et al. 2014; Zhang et al. 2014). In the future, we will optimize the promoter (e.g., U3/U6 promoter from spinach) (Zhou et al. 2018), the distance between two gRNAs (Qi et al. 2016), as well as the protoplast expression system (Oh et al. 2020) to improve the CRIPSR/Cas9 editing efficiency in spinach.

Transcriptome analysis implied the cell wall remodulation in spcsld mutant 
CSLD is essential for the synthesis of polymers, which is critical for the fast-growing primary cell wall at the tip of root hairs (Wang et al. 2001). The root hair tip- localized CSLD3 was predicted to regulate the root hair elongation and cell integrity. In atcs/d3 mutant, the cell wall changed at the site of root hair tip, leading to root hairs elongation was severely inhibited (Wang et al. 2001). Our transcriptomics results revealed large number of genes were involved in cell wall remodulation and membrane trafficking in the Spo23361 mutant. In the mutant, the expression levels of some expansins and xyloglucan endotransglucosylase/hydrolases (XTH) were increased, which could enhance the dissociation of polysaccharide complex and adjustment of xyloglucans, respectively (Cosgrove 2005), therefore resulting in cell wall relaxation and extension. Moreover, the increase of plasma membrane $\mathrm{H}^{+}$-ATPase could facilitate the extracellular acidification activating expansin activity (Cosgrove 2005). Besides, the increased endo- $\beta$-1,3glucanase and $\beta-1,3-1,4-$ glucanase may induce the hydrolysis of the glucan polymer cross-links as well (Thomas et al. 2000; Li et al. 2020). In addition, the decrease of arabinogalactan proteins has potential to reduce the cross-link of pectin and arabinoxylan impairing the wall thickening (Tan et al. 2013; Showalter 2001). All these changes implied that the cell wall loosening was enhanced, while its strength was reduced in mutant. On the other hand, the decease of galacturonosyltransferases (GATL) could affect the pectin synthesis, and the increase of pectin acetylesterase has capacity to induce the cleavage of the acetylester bond from pectin (Kong et al. 2011; Philippe et al. 2017), which might inhibit pectin synthesis and reduce cell wall rigidity in Spo23361 mutant. Importantly, the changes of three lignin synthesis-related genes, encoding cinnamyl alcohol dehydrogenase, hydroxycinnamoyl CoA quinate hydroxycinnamoyl transferase, and laccase, suggest that the disturbance of lignin in cell wall could affect the root hair growth (Van Acker et al. 2017; Bonawitz et al. 2010). Additionally, the enrichment of numbers of genes were involved in various stress responses (e.g., ion ions, hypoxia, and bacterium), signaling, transcription, protein folding and processing, ROS scavenging, and energy metabolism, suggesting that the editing of CSLD gene could result in cell wall change and series modulations in signal transduction, gene expression regulation, and various metabolisms, which may lead to inhibition of root hair growth in Spo23361 mutant.

\section{Conclusion And Prospects}

The present study indicates that the CRISPR/Cas9 via tRNA-sgRNA strategy is a highly efficient tool for genome editing in spinach. Homozygous and bi-allelic mutations were induced at different loci by CRISPR/Cas9 in hairy roots of spinach, which provides a powerful tool and model to study the gene function in spinach roots. Importantly, the CRISPR/Cas9 system has potential to be utilized for investigating the development and stressresponsive mechanism in spinach and breeding novel spinach germplasm with useful agronomic traits.

\section{Declarations}

\section{Acknowledgments}

We are grateful to Pro. Yao-Guang Liu (Huanan Agricultural University, China) for kindly providing the whole set of CRISPR/Cas9 vectors including pYLCRISPR/Cas9 35s-B and pYLsgRNA-AtU3b. We also thank Pro. Ying Lu (Shanghai Ocean University, China) for helping off-target search including program modification and debugger, looking for off-target positions on chromosomes in spinach by blast tool, and off-target sequence extraction. This study was supported by the Foundation of Shanghai Science and Technology Committee, China (No.17391900600) and the Fund of Shanghai Engineering Research Center of Plant Germplasm Resources (No.17DZ2252700) to S.D., the National Nature Science Foundation of China (No.31902012) to M.S, and QIBEBT and Dalian National Laboratory for Clean Energy (DNL), CAS (No. QIBEBT I201921) to C.F.

\section{Author contribution}

SD and CF planned and designed the research; YC, YX, YZ, HZ, ZQ, CB, HZ, DM, and QW performed the research, analyzed, and interpreted data; and YC, MS, CF and SD wrote the manuscript.

\section{Conflict of interests}

No conflict of interest exits in the submission of this manuscript, and manuscript is approved by all authors for publication. The work described was original research that has not been published previously, and not under consideration for publication elsewhere, in whole or in part. All the authors listed have approved the manuscript that is enclosed.

\section{Data availability statement}

All data, models, and code generated or used during the study appear in the submitted article.

\section{References}

1. Bai Z, Li W, Jia Y, Yue Z, Jiao J, Huang W, Xia P, Liang Z (2018) The ethylene response factor SmERF6 co-regulates the transcription of SmCPS1 and SmKSL1 and is involved in tanshinone biosynthesis in Salvia miltiorrhiza hairy roots. Planta. 248:243-255

2. Barrangou R, Marraffini LA. CRISPR-Cas systems: prokaryotes upgrade to adaptive immunity (2014) Mol Cell 54:234-44

3. Bernal AJ, Yoo CM, Mutwil M, Jensen JK, Hou G, Blaukopf C, Sørensen I, Blancaflor EB, Scheller HV, Willats WG (2008) Functional analysis of the cellulose synthase-like genes CSLD1, CSLD2, and CSLD4 in tip-growing Arabidopsis cells. Plant Physiol 148:1238-53 
4. Bonawitz ND, Chapple C (2010) The genetics of lignin biosynthesis: connecting genotype to phenotype. Annu Rev Genet 44:337-63

5. Cai Y, Chen L, Liu X, Sun S, Wu C, Jiang B, Han T, Hou W (2015) CRISPR/Cas9-mediated genome editing in soybean hairy roots. PLoS One. 10: e0136064

6. Cosgrove DJ (2005) Growth of the plant cell wall. Nat Rev Mol Cell Biol 6: 850-61

7. Dong F, Xie K, Chen Y, Yang Y, Mao Y (2017) Polycistronic tRNA and CRISPR guide-RNA enables highly efficient multiplexed genome engineering in human cells. Biochem Biophys Res Commun 482:889-895

8. Fauser F, Schiml S, Puchta H (2014) Both CRISPR/Cas-based nucleases and nickases can be used efficiently for genome engineering in Arabidopsis thaliana. Plant J 79:348-59

9. Favery B, Ryan E, Foreman J, Linstead P, Boudonck K, Steer M, Shaw P, Dolan L (2001) KOJAK encodes a cellulose synthase-like protein required for root hair cell morphogenesis in Arabidopsis. Genes Dev 15:79-89

10. Feng Z, Zhang B, Ding W, Liu X, Yang DL, Wei P, Cao F, Zhu S, Zhang F, Mao Y, Zhu JK (2013) Efficient genome editing in plants using a CRISPR/Cas system. Cell Res 23:1229-32

11. Gagnon JA, Valen E, Thyme SB, Huang P, Akhmetova L, Pauli A, Montague TG, Zimmerman S, Richter C, Schier AF (2014) Efficient mutagenesis by Cas9 protein-mediated oligonucleotide insertion and large-scale assessment of single-guide RNAs. PLoS One 9: e98186

12. Gao C (2021) Genome engineering for crop improvement and future agriculture. Cell 184:1621-1635

13. Ge C, Cai X, Xu C, Wang X, Deng J, Liu S, Zhao Qi, Dai S, Wang Q (2015) A new heat-resistant spinach cultivar 'Hubo 1'. Acta Horti Sinica 42: 399400

14. Georgiev MI, Agostini E, Ludwig-Müller J, Xu J (2012) Genetically transformed roots: from plant disease to biotechnological resource. Trends Biotechnol 30:528-37

15. Jinek M, Chylinski K, Fonfara I, Hauer M, Doudna JA, Charpentier E (2012) A programmable dual-RNA-guided DNA endonuclease in adaptive bacterial immunity. Science 337: 816-21

16. Johnson RA, Gurevich V, Filler S, Samach A, Levy AA (2015) Comparative assessments of CRISPR-Cas nucleases' cleavage efficiency in planta. Plant Mol Biol 87:143-56

17. Kirchner TW, Niehaus M, Debener T, Schenk MK, Herde M (2017) Efficient generation of mutations mediated by CRISPR/Cas9 in the hairy root transformation system of Brassica carinata. PLoS One 12: e0185429

18. Kong Y, Zhou G, Yin Y, Xu Y, Pattathil S, Hahn MG (2011) Molecular analysis of a family of Arabidopsis genes related to galacturonosyltransferases. Plant Physiol 155:1791-805

19. Li B, Cui G, Shen G, Zhan Z, Huang L, Chen J, Qi X (2017) Targeted mutagenesis in the medicinal plant Salvia miltiorrhiza. Sci Rep 7: 43320

20. Li J, Cao C, Jiang Y, Huang Q, Shen Y, Ni J (2020) A Novel Digestive GH16 $\beta$-1,3(4)-Glucanase from the Fungus-Growing Termite Macrotermes barneyi. Appl Biochem Biotechnol 192:1284-1297

21. Li S, Yu J, Li Y, Zhang H, Bao X, Bian J, Xu C, Wang X, Cai X, Wang Q, Wang P, Guo S, Miao Y, Chen S, Qin Z, Dai S (2019) Heat-Responsive Proteomics of a Heat-Sensitive Spinach Variety. Int J Mol Sci. 20: 3872

22. Libault M, Brechenmacher L, Cheng J, Xu D, Stacey G (2010) Root hair systems biology. Trends Plant Sci 15:641-50

23. Liu X, Homma A, Sayadi J, Yang S, Ohashi J, Takumi T (2016) Sequence features associated with the cleavage efficiency of CRISPR/Cas9 system. Sci Rep 6:19675

24. Ma X, Liu YG (2016) CRISPR/Cas9-based multiplex genome editing in monocot and dicot plants. Curr Protoc Mol Biol 115(1): 31.6.1-31.6.21

25. Nakayasu M, Akiyama R, Lee HJ, Osakabe K, Osakabe Y, Watanabe B, Sugimoto Y, Umemoto N, Saito K, Muranaka T, Mizutani M (2018) Generation of a-solanine-free hairy roots of potato by CRISPR/Cas9 mediated genome editing of the St16DOX gene. Plant Physiol Biochem 131:70-77

26. Oh Y, Lee B, Kim H, Kim SG (2020) A multiplex guide RNA expression system and its efficacy for plant genome engineering. Plant Methods 16:37

27. Park S, Szumlanski AL, Gu F, Guo F, Nielsen E (2011) A role for CSLD3 during cell-wall synthesis in apical plasma membranes of tip-growing root-hair cells. Nat Cell Biol 13:973-80

28. Philippe F, Pelloux J, Rayon C (2017) Plant pectin acetylesterase structure and function: new insights from bioinformatic analysis. BMC Genom 18:456

29. Qi G, Hu R, Yu L, Chai G, Cao Y, Zuo R, Kong Y, Zhou G (2013) Two poplar cellulose synthase-like D genes, PdCSLD5 and PdCSLD6, are functionally conserved with Arabidopsis CSLD3. J Plant Physiol 170:1267-76

30. Qi W, Zhu T, Tian Z, Li C, Zhang W, Song R (2016) High-efficiency CRISPR/Cas9 multiplex gene editing using the glycine tRNA-processing system-based strategy in maize. BMC Biotechnol 16:58

31. Ren X, Yang Z, Xu J, Sun J, Mao D, Hu Y, Yang SJ, Qiao HH, Wang X, Hu Q, Deng P, Liu LP, Ji JY, Li JB, Ni JQ (2014) Enhanced specificity and efficiency of the CRISPR/Cas9 system with optimized sgRNA parameters in Drosophila. Cell Rep 9:1151-62

32. Richmond TA, Somerville CR (2000) The cellulose synthase superfamily. Plant Physiol 124: 495-498 
33. Ron M, Kajala K, Pauluzzi G, Wang D, Reynoso MA, Zumstein K, Garcha J, Winte S, Masson H, Inagaki S, Federici F, Sinha N, Deal RB, BaileySerres J, Brady SM (2014) Hairy root transformation using Agrobacterium rhizogenes as a tool for exploring cell type-specific gene expression and function using tomato as a model. Plant Physiol 166:455-469

34. Shan Q, Wang Y, Li J, Zhang Y, Chen K, Liang Z, Zhang K, Liu J, Xi JJ, Qiu JL, Gao C (2013) Targeted genome modification of crop plants using a CRISPR-Cas system. Nat Biotechnol 31:686-8

35. Showalter AM (2001) Arabinogalactan-proteins: structure, expression and function. Cell Mol Life Sci 58:1399-1417

36. Tan L, Eberhard S, Pattathil S, Warder C, Glushka J, Yuan C, Hao Z, Zhu X, Avci U, Miller JS, Baldwin D, Pham C, Orlando R, Darvill A, Hahn MG, Kieliszewski MJ, Mohnen D (2013) An Arabidopsis cell wall proteoglycan consists of pectin and arabinoxylan covalently linked to an arabinogalactan protein. Plant Cell 25:270-87

37. Thomas BR, Inouhe M, Simmons CR, Nevins DJ (2000) Endo-1,3;1,4-beta-glucanase from coleoptiles of rice and maize: role in the regulation of plant growth. Int J Biol Macromol 27:145-9

38. Van Acker R, Déjardin A, Desmet S, Hoengenaert L, Vanholme R, Morreel K, Laurans F, Kim H, Santoro N, Foster C, Goeminne G, Légée F, Lapierre C, Pilate G, Ralph J, Boerjan W (2017) Different routes for conifer- and sinapaldehyde and higher saccharification upon deficiency in the dehydrogenase CAD1. Plant Physiol 175:1018-1039

39. Wang P, Zhang J, Sun L, Ma Y, Xu J, Liang S, Deng J, Tan J, Zhang Q, Tu L, Daniell H, Jin S, Zhang X (2018) High efficient multisites genome editing in allotetraploid cotton (Gossypium hirsutum) using CRISPR/Cas9 system. Plant Biotechnol J 16:137-150

40. Wang W, Wang L, Chen C, Xiong G, Tan XY, Yang KZ, Wang ZC, Zhou Y, Ye D, Chen LQ (2011). Arabidopsis CSLD1 and CSLD4 are required for cellulose deposition and normal growth of pollen tubes. J Exp Bot. 62:5161-77

41. Wang X, Cnops G, Vanderhaeghen R, De Block S, Van Montagu M, Van Lijsebettens M (2001) AtCSLD3, a cellulose synthase-like gene important for root hair growth in arabidopsis. Plant Physiol 126:575-86

42. Xie K, Minkenberg B, Yang Y (2015) Boosting CRISPR/Cas9 multiplex editing capability with the endogenous tRNA-processing system. Proc Natl Acad Sci U S A 112: 3570-3575

43. Xu C, Jiao C, Sun H, Cai X, Wang X, Ge C, Zheng Y, Liu W, Sun X, Xu Y, Deng J, Zhang Z, Huang S, Dai S, Mou B, Wang Q, Fei Z, Wang Q (2017) Draft genome of spinach and transcriptome diversity of 120 Spinacia accessions. Nat Commun 8:15275

44. Xu Y, Cao YP, Wang Y, Fu C, Dai S (2019) Agrobacterium rhizogenes-mediated transformation system of Spinacia oleracea. Chin Bull Bot (4), 515 $-521$.

45. Yin L, Verhertbruggen Y, Oikawa A, Manisseri C, Knierim B, Prak L, Jensen JK, Knox JP, Auer M, Willats WG, Scheller HV (2011) The cooperative activities of CSLD2, CSLD3, and CSLD5 are required for normal Arabidopsis development. Mol Plant 4:1024-1037

46. Yoo CM, Quan L, Blancaflor EB (2012) Divergence and redundancy in cs/d2 and cs/d3 function during Arabidopsis thaliana root hair and female gametophyte development. Front Plant Sci 3, 111

47. Zhang H, Zhang J, Wei P, Zhang B, Gou F, Feng Z, Mao Y, Yang L, Zhang H, Xu N, Zhu JK (2014) The CRISPR/Cas9 system produces specific and homozygous targeted gene editing in rice in one generation. Plant Biotechnol J 12:797-807

48. Zhao Q, Chen W, Bian J, Xie H, Li Y, Xu C, Ma J, Guo S, Chen J, Cai X, Wang X, Wang Q, She Y, Chen S, Zhou Z, Dai S (2018) Proteomics and phosphoproteomics of heat stress-responsive mechanisms in spinach. Front Plant Sci 9:800.

49. Zhou Z, Tan H, Li Q, Chen J, Gao S, Wang Y, Chen W, Zhang L (2018) CRISPR/Cas9-mediated efficient targeted mutagenesis of RAS in Salvia miltiorrhiza. Phytochemistry 148:63-70

50. Zhu H, Li C, Gao C (2020) Applications of CRISPR-Cas in agriculture and plant biotechnology. Nat Rev Mol Cell Biol (11): 661-677

\section{Figures}


a
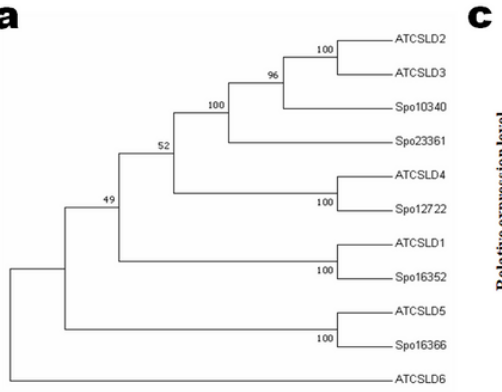

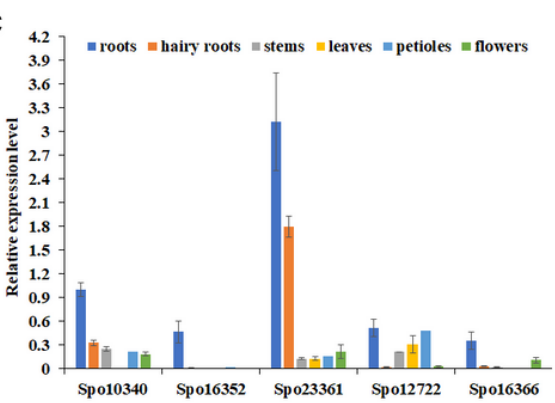

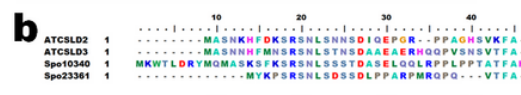

$\begin{array}{lllll}\text { Sp010340 } & \text { Spo16352 } & \text { Spo23361 } & \text { Sp012722 } & \text { Spo16366 }\end{array}$

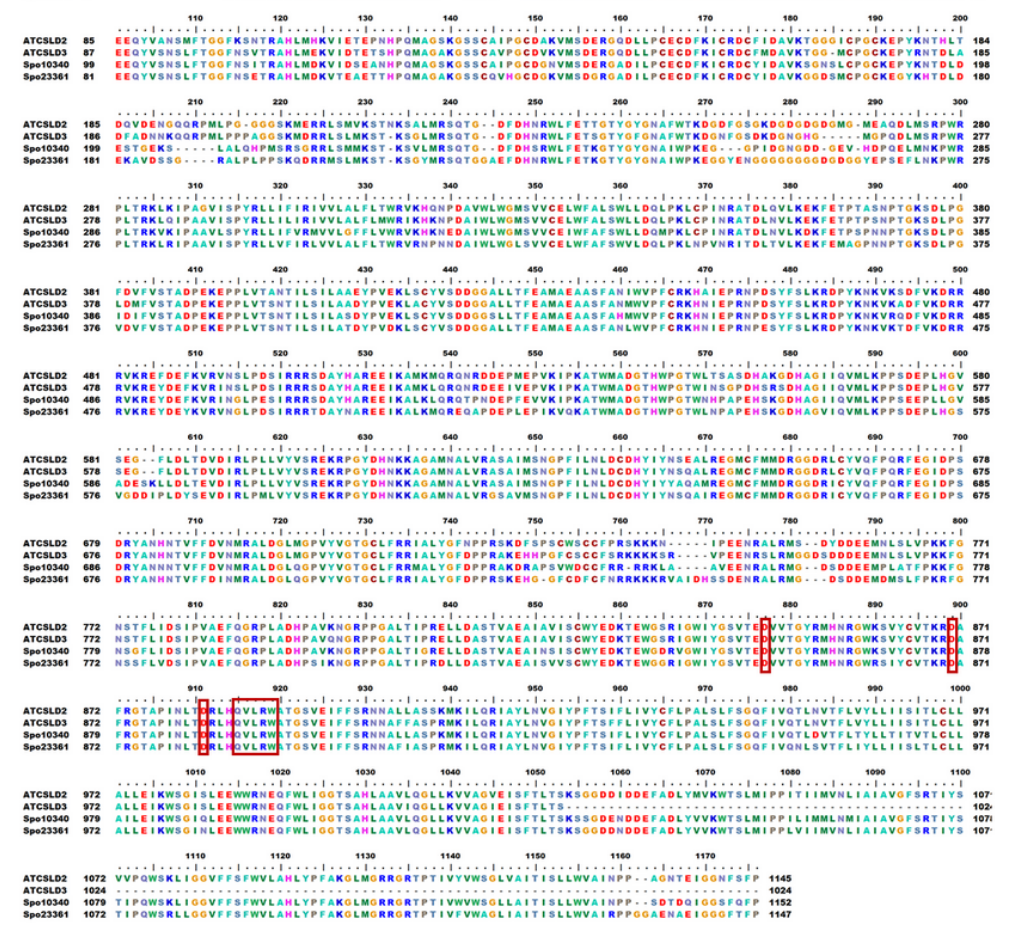

Figure 1

Bioinformatic analysis of Spo23361 and Spo10340 and expression analysis of the five predicted CSLD genes using qRT-PCR. (a) Joined phylogenetic tree of CSLD members in spinach and Arabidopsis constructed with MEGA 4.0 by the Neighbor-Joining (NJ) method with 1000 bootstrap replicates. (b) Amino acid sequence alignment of Spo23361, Spo10340, AtCSLD2 and AtCSLD3. The identical and similar amino acid residues are present with same color, respectively. The conserved motif is labeled with red frame. (c) qRT-PCR detection of the expression of five predicted genes, Spo23361, Spo10340, Spo12722, Spo16352, Spo16366, in the stem, leaf, flower, stipe, root and hairy root of spinach. The relative mRNA abundance of each gene in all samples was normalized with respect to reference gene Spo23599, an actin gene of spinach. 

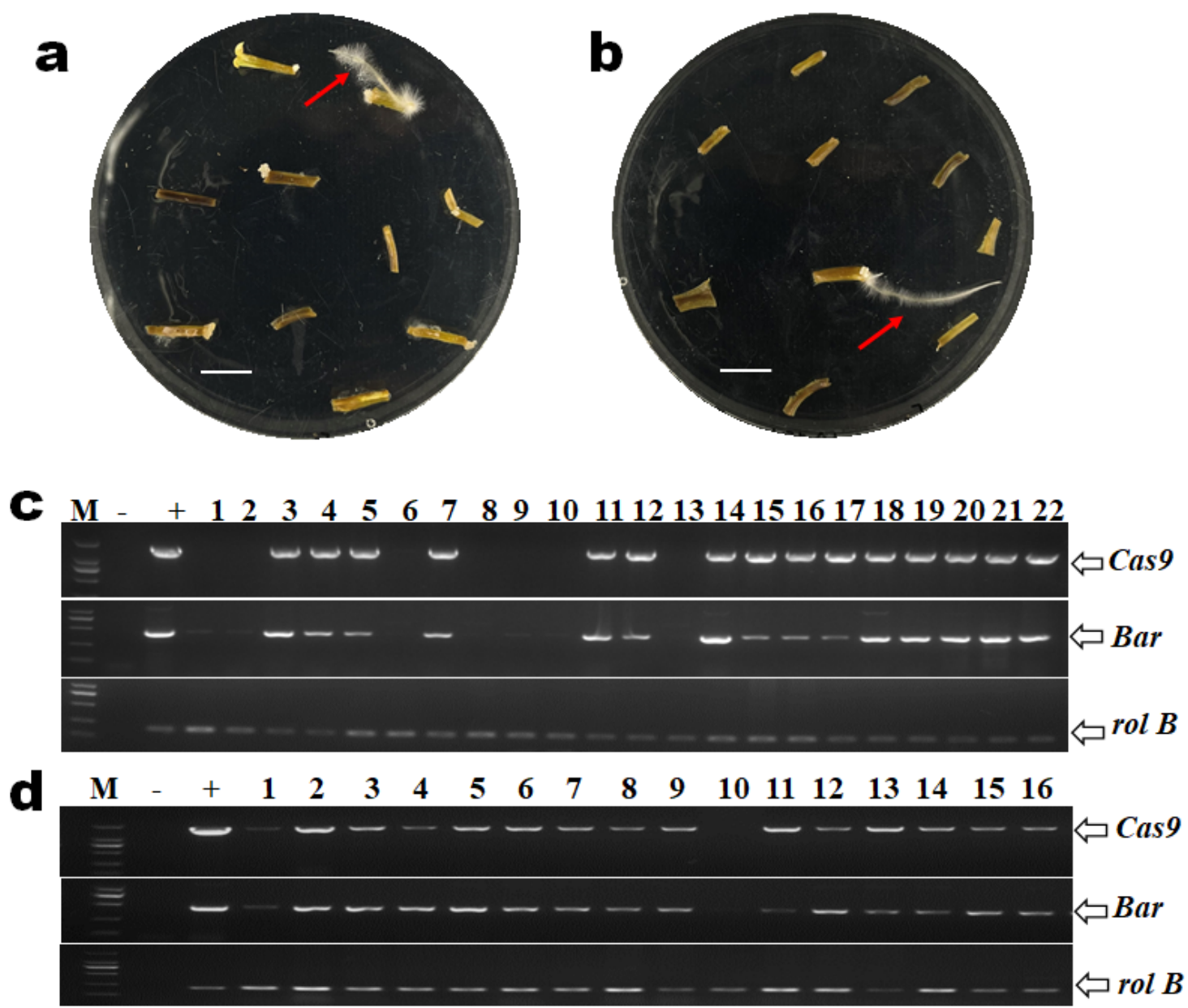

\section{Figure 2}

Identification of co-transformed hairy root lines of spinach induced by A. rhizogenes strain LBA9402. (a) and (b) Hairy roots appeared after 10 days of infection (a) and 20 days of infection (b). (c) and (d) Identification of co-transformed hairy root lines of Spo10340 (c) and Spo23361 (d) by genome Polymerase Chain Reaction (PCR) screening. The hairy root lines that could amplified the three genes (i.e., Bar, Cas 9 and ro/B) were considered to be the co-transformed lines. 15 lines were obtained form 22 hairy root lines of Sp10340, and 15 lines were identified among 16 hairy root lines of Spo23361. Untransformed roots served as a negative control, and A. rhizogenes strain LBA9402 harboring the CRISPR/Cas9 plasmid was used as a positive control. The expected bands sizes of the Bar, Cas 9 and $r o l B$ are indicated by arrows. 

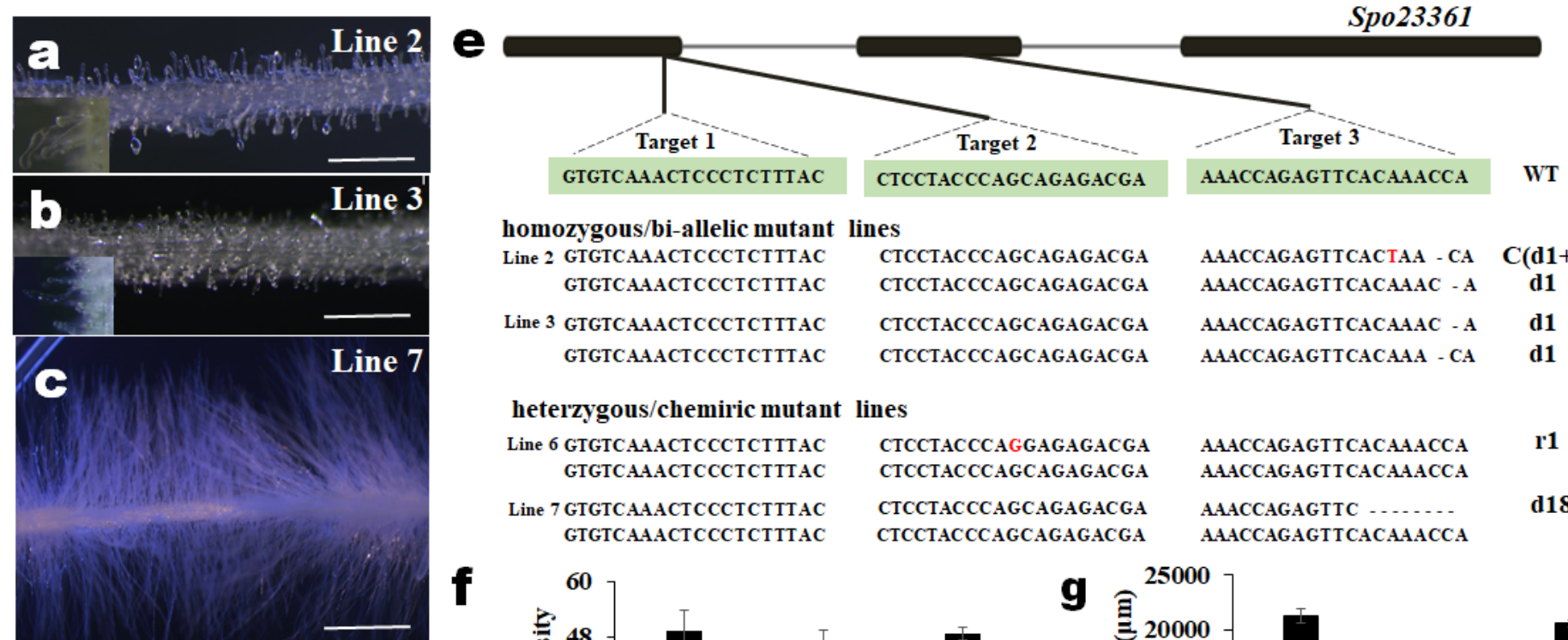

homozygous/bi-allelic mutant lines

Line 2 GTGTCAAACTCCCTCTTTAC CTCCTACCCAGCAGAGACGA GTGTCAAACTCCCTCTTTAC CTCCTACCCAGCAGAGACGA

AAACCAGAGTTCACTAA - CA AAACCAGAGTTCACAAAC - A

Line 3 GTGTCAAACTCCCTCTTTAC CTCCTACCCAGCAGAGACGA AAACCAGAGTTCACAAAC - A GTGTCAAACTCCCTCTTTAC CTCCTACCCAGCAGAGACGA

AAACCAGAGTTCACAAA - CA

d1

d1

heterzygous/chemiric mutant lines

Line 6 GTGTCAAACTCCCTCTTTAC CTCCTACCCAGGAGAGACGA GTGTCAAACTCCCTCTTTAC CTCCTACCCAGCAGAGACGA

AAACCAGAGTTCACAAACCA AAACCAGAGTTCACAAACCA Line 7 GTGTCAAACTCCCTCTTTAC CTCCTACCCAGCAGAGACGA

AAACCAGAGTTC $\ldots . . . .$. GTGTCAAACTCCCTCTTTAC CTCCTACCCAGCAGAGACGA
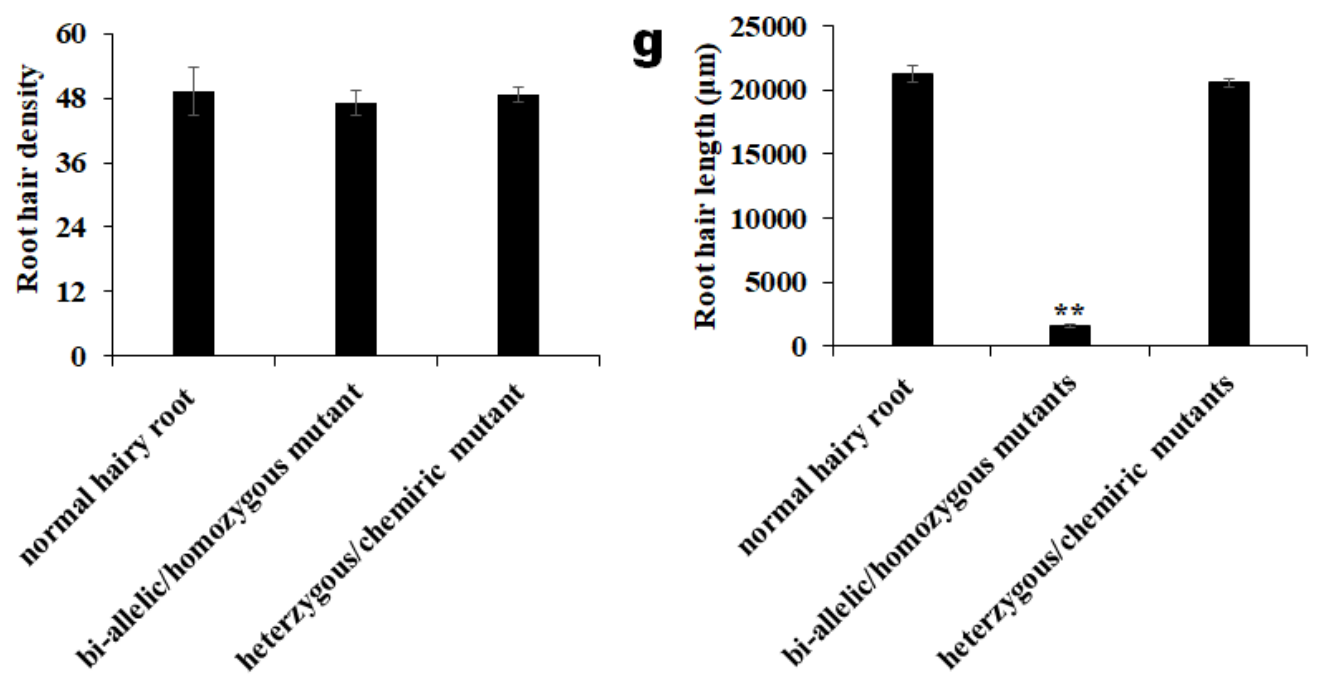

Figure 3

Phenotyping and genotyping of edited hairy root lines of Spo23361. (a) and (b) The phenotype of the bi-allelic mutated line, Line 2 (a) and Line 3 (b) of Spo23361. Bar $\nabla 4 \mu \mathrm{m}$. (c) The phenotype of the heterozygous mutated line 7 of Spo23361. Bar $\otimes 20 \mu m$. (d) The phenotype of the normal hairy root. Bar $\nabla 20 \mu \mathrm{m}$. (e) The mutation types of the line 2, line 3 line 6 and line 7. The mutation types of each target were labeled, respectively. Deletions

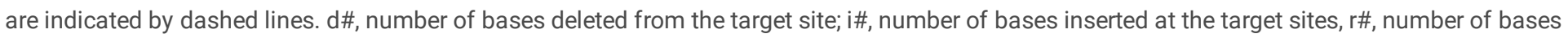
replaced at the target site. The replaced bases were in red and the inserted bases were in green. (f) Root hair density and ( $\mathrm{g}$ ) root hair length analysis of normal hairy roots, bi-allelic/homozygous mutated and heterozygous/chimeric mutated hairy root lines. Root hair length and density were measured at the region 1.5 to $2 \mathrm{~mm}$ from the root tip. Root hair density is shown as root hair number per $\mathrm{mm}^{2} .24$ roots of three lines ( 8 roots for each line) were measured for each mutation type, values were mean \pm SD. Asterisk ( $\left.{ }^{\star *}\right)$ indicates that difference is significant at $p<0.01$. 


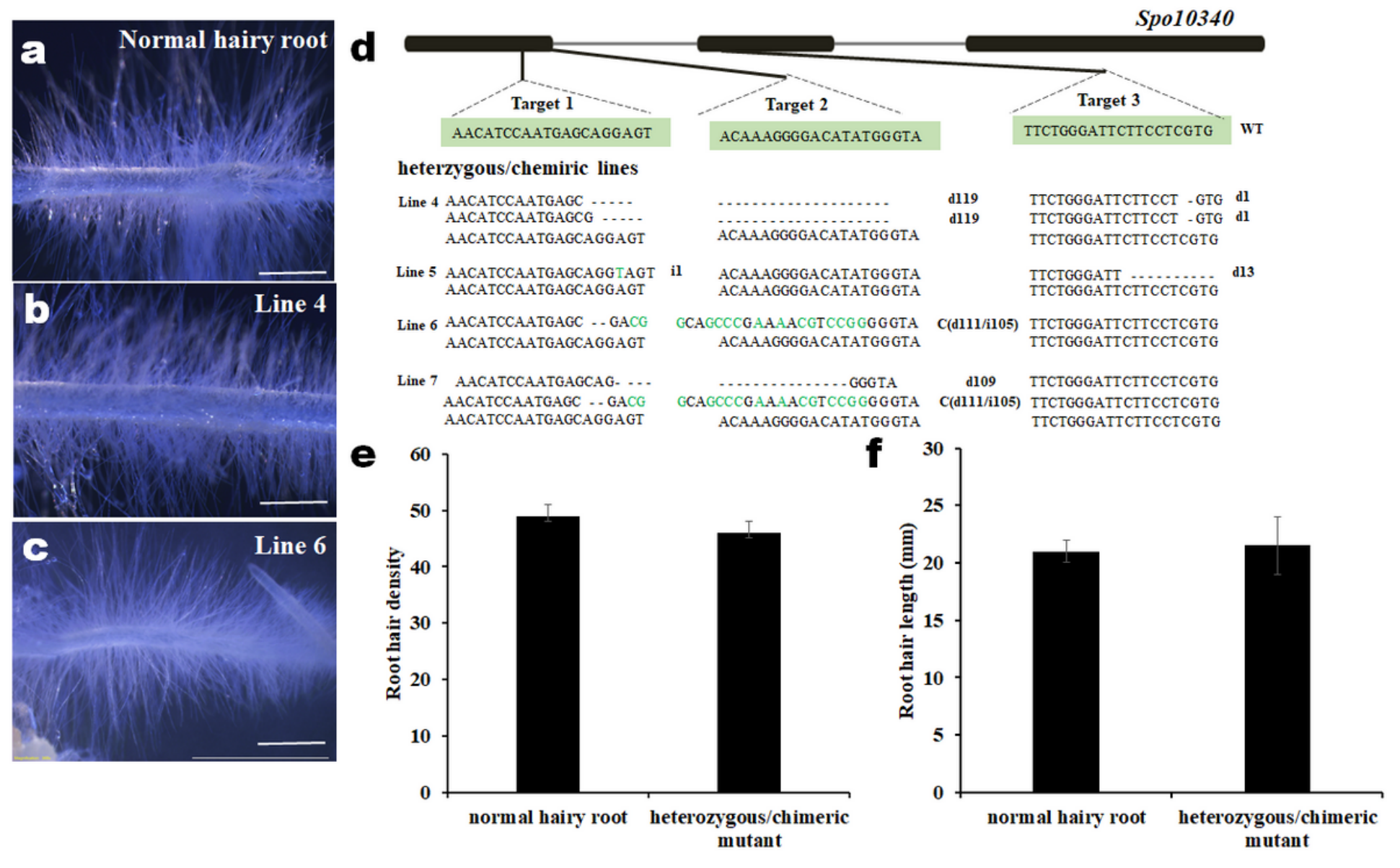

Figure 4

Phenotyping and genotyping of edited hairy root lines of Spo10340. (a), (b) and (c) The root hair phenotype of normal hairy root (a), line 4 (chimeric line) (b) and line 6 (heterozygous line) (c) of Spo10340. Bar $₫ 20 \mu \mathrm{m}$. (d) The mutation types of the line 4, line 5, line 6 and line 7 (heterzygous

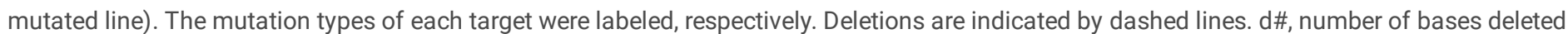
from the target site; i\#, number of bases inserted at the target site; $r \#$, number of bases replaced at the target site. The replaced bases were in red and the inserted bases were in green. (e) Root hair density and (f) root hair length analysis of normal hairy roots and heterozygous/chimeric mutated hairy root lines. Root hair length and density were measured at the region 1.5 to $2 \mathrm{~mm}$ from the root tip. Root hair density is shown as root hair number per $\mathrm{mm}^{2} .24$ roots of three lines (8 roots for each line) were measured for each mutation type, values were mean \pm SD. 

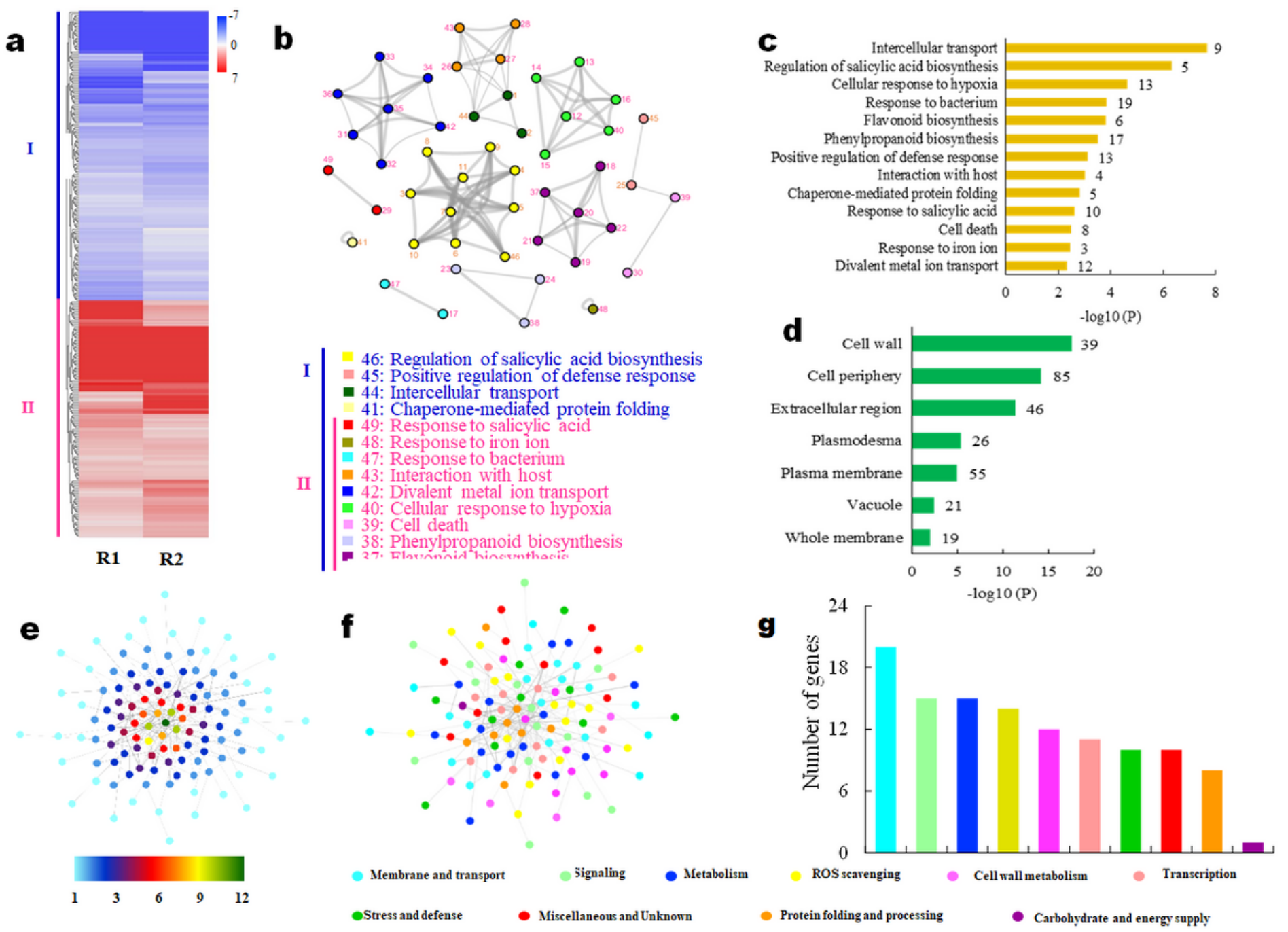

Figure 5

Cluster analysis and enrichment of differential expression genes, and the predicted interaction networks of their encoding proteins in Spo23361. (a) Hierarchical clustering analysis of differential expression genes. Two columns represent two replications of fold change (Log2 transformed) of DEGs in Spo23361 mutant (R1, R2). The rows represent individual genes. The detailed information is listed in Table S3. The increased or decreased genes are indicated in red or blue, respectively. (b) Network of enriched terms of GO biological processes and KEGG pathways across the DEG lists of the two clusters. The network is visualized using Cytoscape software, where each node represents an enriched term. The term genes next to the nodes are shown in Table S4. The terms with a similarity $>0.3$ are connected by edges. The same color nodes represent a subset of enriched terms, where the terms with the best p-values were highlighted with bold words, and is according to the GO biological process or KEGG pathway shown under the network. The color of the term genes (i.e., blue and pink) are corresponded to the Cluster I and Cluster II, respectively. (c) Bar graph shown the summary of subsets enriched according to GO biological processes and KEGG pathways. The number in right represents number of DEGs enriched in each subset. (d) Enrichment analysis for cellular components of DEGs by GO annotation. The number in right represents number of DEGs enriched in each term. (e-f) Predicted interaction networks of proteins encoding by differentially-expressed genes. The protein-protein interaction was predicted using STRING 10 and visualized by Cytoscape software (Version 3.7.1). Proteins (nodes) were shown as bubbles filled with different colors. The related interactions (edges) between proteins were shown as gray lines. (e) Heatmaps representing the node degree of each protein in the interaction network, respectively. The range of degree distribution is shown as a color gradient from light blue to dark green. (f) Proteins encoding by differentially-expressed genes in various function categories, which were indicated by the node fill color. (g) Numbers of proteins encoding by differentially-expressed genes in different function categories.

\section{Supplementary Files}

This is a list of supplementary files associated with this preprint. Click to download. 
- 05TableS3ZH20210806.xlsx

- 06TableS4ZH20210501.xlsx

- 07TableS5ZH20210501.xlsx

- 08TableS6ZH20210501.xlsx

- 09TableS7ZH20210806.xlsx

- 10TableS820210806.docx

- 11FigureS120211216.docx 